\title{
Singlet and Triplet Exciton Dynamics of Violanthrone
}

\author{
Elham M. Gholizadeh ${ }^{1}$, Shyamal K. K. Prasad ${ }^{1}$, Lara Gillan ${ }^{2}$, \\ Dane R. McCamey ${ }^{2}$, Murad J. Y. Tayebjee ${ }^{3}$ and Timothy W. Schmidt ${ }^{1 *}$ \\ ${ }^{1}$ ARC Centre of Excellence in Exciton Science, \\ School of Chemistry, UNSW Sydney, NSW 2052, Australia \\ ${ }^{2}$ ARC Centre of Excellence in Exciton Science, \\ School of Physics, UNSW Sydney, NSW 2052, Australia \\ ${ }^{3}$ School of Photovoltaic and Renewable Energy Engineering, UNSW Sydney, NSW 2052, Australia
}

*To whom correspondence should be addressed; E-mail: timothy.schmidt@ unsw.edu.au.

\begin{abstract}
The exciton dynamics of violanthrone-79 are investigated in solution and in the solid state. In solution, the photo-prepared singlet is found to exhibit a strong ground-state bleach and stimulated emission feature, but when sensitized in its triplet state, exhibits only a narrow and weak ground-state bleach. As supported by density functional theory calculations, this is explained by the triplet state having absorptions in the same region, with a similar oscillator strength, as the ground state molecule. In solid films, the excited singlet is found to survive only $100 \mathrm{ps}$, giving way to a long-lived transient absorption spectrum with characteristics reminiscent of the triplet in solution. This is interpreted in terms of singlet fission in the solid film.
\end{abstract}

Excitonic interactions involving the interplay of excited singlet excitons and pairs of triplet excitons in organic semiconductors have the potential to transform a number of technologies, including solar photovoltaics,[1-7] photocatalysis,[8, 9] photodynamic therapy,[10] and biological imaging.[11] The conversion of triplet excitons generated with low energy photons into a higher energy singlet excitons is known as triplet fusion, or photochemical upconversion.[1, 8, 12-15] The excitonic interaction at play, triplet-triplet annihilation, is the conjugate process to 
singlet fission.[16, 17] In singlet fission, a high-energy singlet state splits into two lower-energy triplet states, with the latter being available for energy harvesting.[3, 4, 18-21] As such, in a photovoltaic device, high energy photons generate double the photocurrent of lower energy photons, raising the efficiency limit from $33.7 \%$ to $45.9 \%$ under one sun illumination.[22]

To explore the low-energy limits of triplet fusion, chromophores with low-lying triplets are required. Recently, we demonstrated that the dye violanthrone-79 (V79) could be used as an annihilator to achieve photochemical upconversion from below the silicon band gap.[23, 24] However, implementing this as a solid state technology is hindered by the poor performance of V79 in the solid state where it has a very low fluorescence yield. The related molecule, violanthrone78, is known to have an excited-state lifetime in the solid state as short as $20 \mathrm{ps,} \mathrm{and} \mathrm{exhibits}$ J-like excitonic coupling.[25] This short lifetime may be due to singlet fission, as the triplet level of V79 is known to be below $0.98 \mathrm{eV}$, and the $0-0$ emission is at $1.85 \mathrm{eV}$.[24] There is precedence for endothermic fission in films of molecules used for triplet fusion in solution. Rubrene, a well-known annihilator used in triplet-triplet annihilation, is known to undergo endothermic singlet fission in the condensed phase, preventing high triplet fusion yields. Doping the material to trap the excited singlet state has been employed to circumvent this problem.[26] However, the details of crystal structure can also play a role in preventing fission.[27]

In this study, we explore the exciton dynamics of V79 using transient absorption spectroscopy, first in solution, and then in a film. The solution triplet state spectrum is revealed by sensitization with platinum octaethylporphyrin (PtOEP). The transient spectrum of a film of V79 reveals a short-lived excited state which gives rise to a longer-lived state. We interpret this behaviour in terms of singlet fission, guided by density functional theory calculations. 


\section{Experimental Methods}

\subsection{Sample Preparation}

Solutions were made in toluene in a $1 \mathrm{~mm}$-pathlength cuvette. V79 was prepared at a maximum optical density of $0.3\left(0.1 \mathrm{mM}, \epsilon_{635}=27600 \mathrm{M}^{-1} \mathrm{~cm}^{-1}\right)$. The peak optical densities of V79 and $\operatorname{PtOEP}\left(\epsilon_{536}=63760 \mathrm{M}^{-1} \mathrm{~cm}^{-1}[28]\right)$ in the mixture were 0.8 and 0.7 respectively $(0.3 \mathrm{mM}$ and $0.1 \mathrm{mM})$. The V79 film was prepared by drop-casting a solution of V79 in toluene onto a glass slide.

\subsection{Spectroscopy}

Transient absorption spectroscopy was performed using a home-built set-up. The probe pulse was generated by focusing the $785 \mathrm{~nm}$ pulse $(1000 \mathrm{~Hz}$, Clark-MXR CPA 2210, $150 \mathrm{fs}$, Ti:sapph. regen. amp.) onto an yttrium aluminium garnet crystal. The subsequent supercontinuum was dispersed by a home-built prism spectrometer, thus allowing simultaneous collection across the visible and near-infrared regions. For fs-ns time-scales, the pump pulse was generated by a 2-stage optical parametric amplifier (TOPAS-C, light conversion). The pump pulses were delayed using a motorized delay stage (Thorlabs, LTS300). An optical chopper modulated the pump laser, blocking every second pulse. For longer $>$ ns time-scales, the pump pulse was generated with the $532 \mathrm{~nm}$ output of a 800 ps pulse-width Nd:YAG laser (InnoLas picolo), with the timing controlled electronically.

The supercontinuum spectrum (probe) and pump pulse energy was measured on a shot-toshot basis. The probe spectrum was converted to differential transmission by comparison of the shots collected respectively with and without the pump pulse. Data points more than 2.5 standard deviations from the mean were removed. The remaining shots were averaged. The final time-wavelength data-set was generated from multiple data points at each pump-probe delay. 


\subsection{Magnetic Field Effects (MFE)}

The photoluminescence intensity of V79 films were measured as a function of magnetic field strength using the method previously reported.[29] Briefly, the sample was placed in the field of an electromagnet (Magnetech MFG-6-24) and irradiated with a $532 \mathrm{~nm}$ diode laser (Thorlabs). The field was calibrated using a gauss meter (Lakeshore 475 DSP), with the applied current controlled by a Keithley 2230G-30-A power supply. The photoluminescence was detected using a diode array detector (Ocean Optics FLAME-S-XR1-ES).

\subsection{Photoconductivity}

Photoconductivity of drop-cast, room temperature V79 film was investigated using our homebuilt microwave absorption apparatus, detailed elsewhere.[30] Briefly, we monitored the change in reflected microwave power as a function of time after photoexcitation with a pulsed laser (Opolette 355).

\section{Computational Methods}

The structure of V79 was approximated as the methyl di-ester, the long hydrocarbon chains not being expected to impact the spectroscopic properties. The equilibrium geometries in the ground and lowest triplet states were obtained using the Gaussian 16 program,[31] at the $\omega B 97 x-D / 6-31 G(d)$ level of theory.[32, 33] At these geometries, vertical spectroscopic transitions were investigated by performing time-dependent density functional theory with the 6$311 \mathrm{G}(\mathrm{d})$ basis set. 


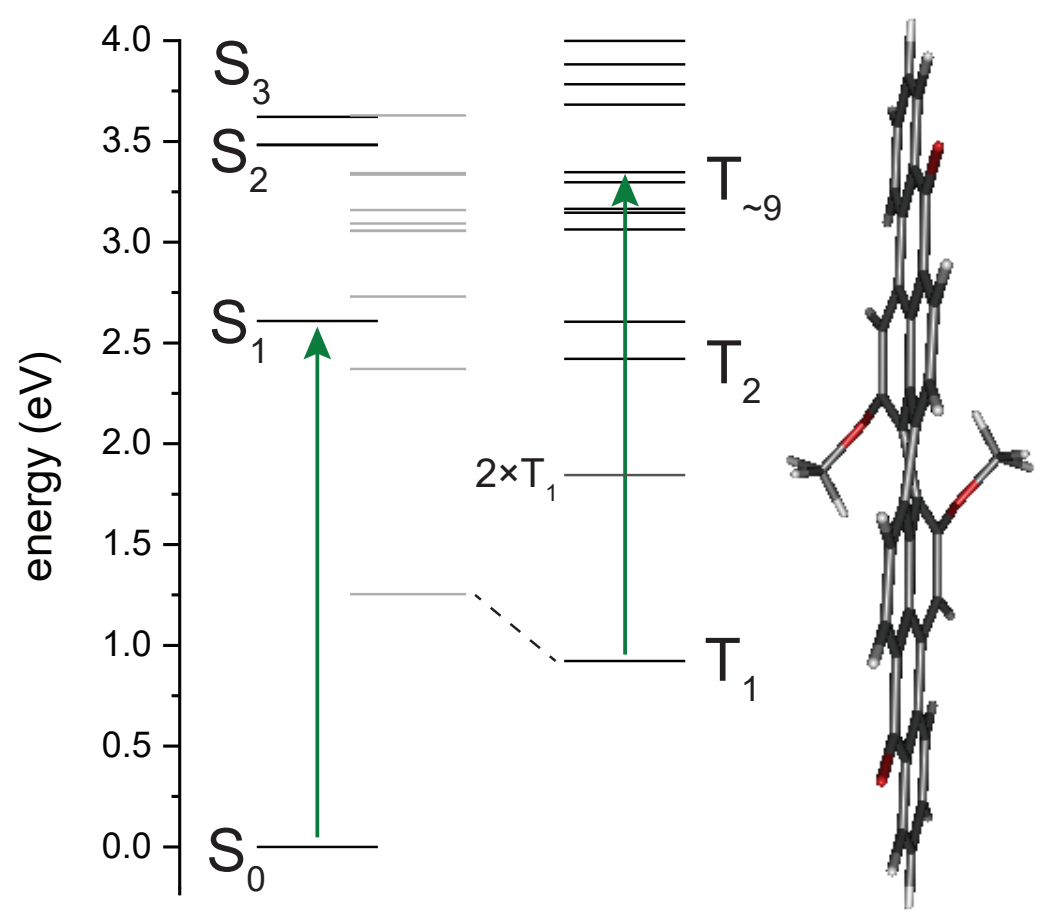

Figure 1: Left: Jablonski diagram calculated by TD-DFT. The relaxed $T_{1}$ energy is set empirically. The strong absorptions are indicated with vertical arrows. Right: The $S_{0} \omega \mathrm{B} 97 \mathrm{X}-\mathrm{D} / 6-$ $31 \mathrm{G}(\mathrm{d})$ geometry ( $C_{2}$ symmetry). 


\section{Results}

\subsection{TD-DFT Calculations}

The results of the TD- $\omega \mathrm{B} 97 \mathrm{X}-\mathrm{D} / 6-311 \mathrm{G}(\mathrm{d}) / / \omega \mathrm{B} 97 \mathrm{X}-\mathrm{D} / 6-31 \mathrm{G}(\mathrm{d})$ calculations are reported in Table 1. At the ground state geometry ( $S_{0}$, depicted in Figure 1), a strong transition at $2.6 \mathrm{eV}$ with $f=0.85$ is calculated to $S_{1}$, polarized perpendicularly to the $C_{2}$ symmetry axis. The unrelaxed $T_{1}$ state is computed by TD-DFT to be at $1.25 \mathrm{eV}$. A large number of triplet states are calculated, but notably at the relaxed $T_{1}$ geometry, a strong transition at $2.4 \mathrm{eV}$ is calculated with $f=0.82$, polarized in the same sense as the $S_{1} \leftarrow S_{0}$ transition. The upper state of this transition is calculated to be $T_{9}$, but is more rigorously denoted $4^{3} \mathrm{~A}$.

The steady-state absorption spectrum of V79 is shown as the dashed curve in Figure 2. The strong absorption of V79 which corresponds to the predicted strong $S_{1} \leftarrow S_{0}$ transition calculated to be at $2.6 \mathrm{eV}$ is found at $2.0 \mathrm{eV}$. The breadth of the absorption band indicates that some of the disparity is due to a change in geometry between $S_{0}$ and $S_{1}$. Nevertheless, as will become apparent, it is important to note that TD-DFT predicts that both the $S_{0}$ and $T_{1}$ states exhibit transitions of similar strength at similar energies.

\subsection{Single-component transient absorption}

The transient absorption (TA) spectrum of dilute V79 in toluene was fit with a single exponential decay. The resulting spectrum is displayed in Figure 2. The comparison to the steady-state absorption indicates a stimulated emission feature at $1.84 \mathrm{eV}$ and a broad ground-state bleach from about $1.9 \mathrm{eV}$ upwards. A notable excited-state absorption is apparent at $1.11 \mathrm{eV}$. The decay was fit to a time constant of 4.97(17) ns.

The TA trace of dilute PtOEP in toluene is fit to a single spectrum, displayed in Figure 2. The standout feature is the bleach, which closely resembles the steady-state absorption spectrum. From the height of the bleach at $2.32 \mathrm{eV}$, we can calibrate the TA spectrum, resulting in an 
Table 1: Transitions of the Violanthrone Chromophore Calculated at the TD- $\omega$ B97x-D/6-

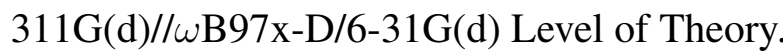

\begin{tabular}{|c|c|c|c|c|c|}
\hline Lower State & Upper State & $\Gamma_{\mu}$ & Energy (eV) & Wavelength (nm) & $\overline{f f}$ \\
\hline \multirow[t]{14}{*}{$S_{0}$} & $T_{1}$ & $\mathrm{~B}$ & 1.2536 & 989 & - \\
\hline & $T_{2}$ & A & 2.3716 & 523 & - \\
\hline & $S_{1}$ & B & 2.6088 & 475 & 0.854 \\
\hline & $T_{3}$ & B & 2.7309 & 454 & - \\
\hline & $T_{4}$ & B & 3.0564 & 406 & - \\
\hline & $T_{5}$ & A & 3.0572 & 406 & - \\
\hline & $T_{6}$ & A & 3.0934 & 401 & - \\
\hline & $T_{7}$ & B & 3.1600 & 392 & - \\
\hline & $T_{8}$ & B & 3.3350 & 372 & - \\
\hline & $T_{9}$ & A & 3.3422 & 371 & - \\
\hline & $S_{2}$ & A & 3.4818 & 356 & 0.000 \\
\hline & $S_{3}$ & B & 3.4845 & 356 & 0.003 \\
\hline & $S_{4}$ & A & 3.6220 & 342 & 0.021 \\
\hline & $T_{10}$ & A & 3.6295 & 342 & - \\
\hline \multirow[t]{12}{*}{$T_{1}$} & $T_{2}$ & $\mathrm{~B}$ & 1.4989 & 827 & 0.017 \\
\hline & $T_{3}$ & A & 1.6830 & 737 & 0.001 \\
\hline & $T_{4}$ & B & 2.1407 & 579 & 0.036 \\
\hline & $T_{5}$ & B & 2.2239 & 558 & 0.0002 \\
\hline & $T_{6}$ & A & 2.2244 & 557 & 0.0002 \\
\hline & $T_{7}$ & A & 2.2427 & 553 & 0.045 \\
\hline & $T_{8}$ & A & 2.3752 & 522 & 0.047 \\
\hline & $T_{9}$ & B & 2.4251 & 511 & 0.820 \\
\hline & $T_{10}$ & A & 2.7604 & 449 & 0.025 \\
\hline & $T_{11}$ & B & 2.8616 & 433 & 0.187 \\
\hline & $T_{12}$ & B & 2.9601 & 419 & 0.0001 \\
\hline & $T_{13}$ & A & 3.0761 & 403 & 0.014 \\
\hline
\end{tabular}



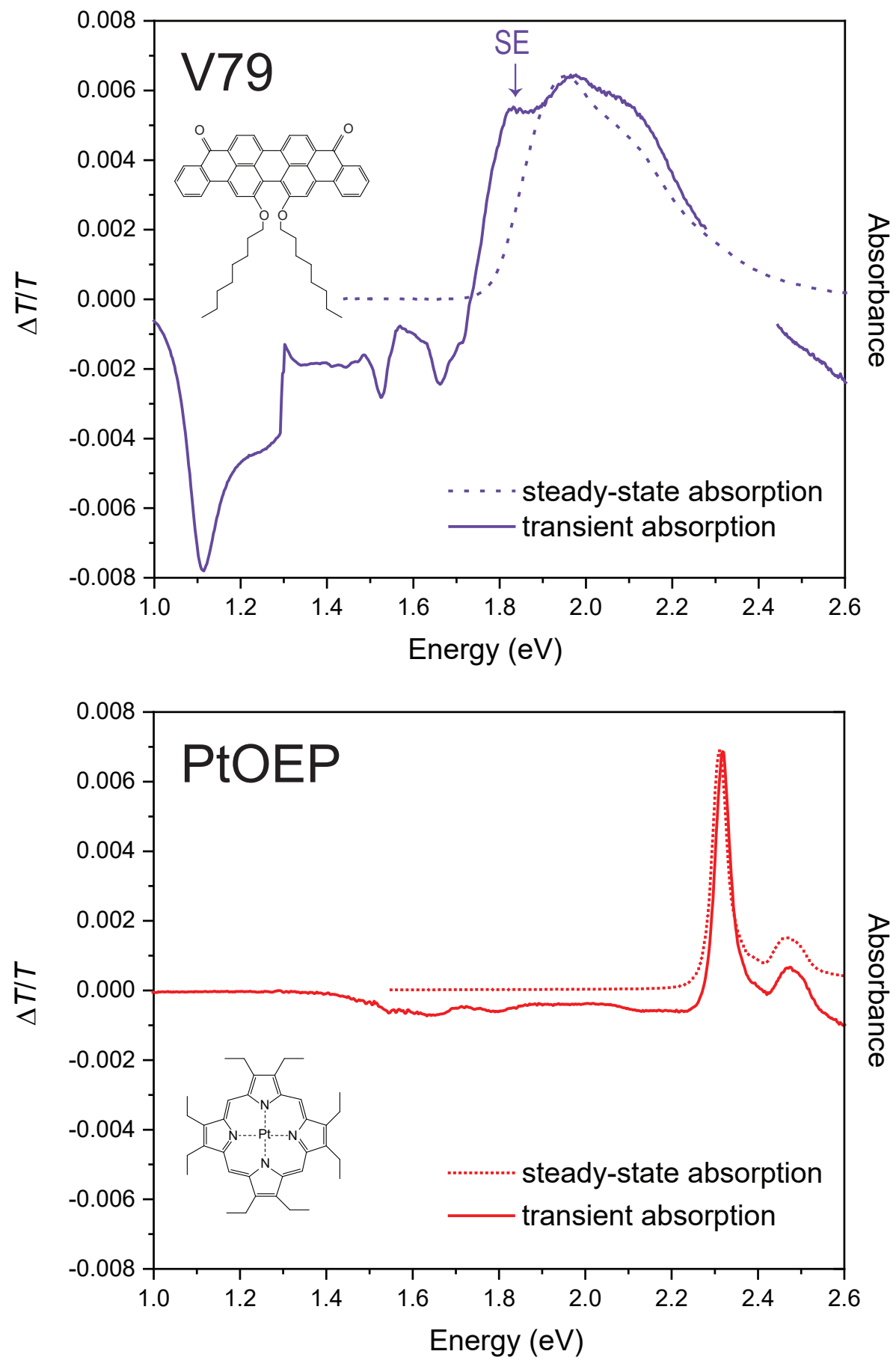

Figure 2: The transient spectra of ${ }^{1} \mathrm{~V} 79$ and ${ }^{3} \mathrm{PtOEP}$, compared to their steady-state absorption spectra (dashed lines). V79 clearly exhibits a stimulated emission (SE) feature at $1.84 \mathrm{eV}$. 
absorption coefficient at $2.48 \mathrm{eV}$ of $572 \mathrm{M}^{-1}$ (1 mm pathlength). A fit to the TA spectrum reveals a lifetime of $338 \mu \mathrm{s}$, which is therefore attributed to ${ }^{3} \mathrm{PtOEP}$. This accords with the absence of a stimulated emission feature.

\subsection{Triplet Sensitization}

The TA spectrum of the mixture of V79 and PtOEP in toluene is displayed in Figure 3. The data set was fit, resulting in decay-associated spectra corresponding to time constants of $6.7 \mathrm{~ns}$, $1.04 \mu \mathrm{s}$ and $16.1 \mu \mathrm{s}$. Both species are excited by the $532 \mathrm{~nm}$ laser pulse. The first spectrum is identified with the excited singlet of V79. It does not exhibit any spectral correlations with the other components, and thus may be considered kinetically unconnected to the other species.

The spectrum of the $1.04 \mu$ s decay is anticorrelated with the spectrum of the $16 \mu$ s decay in the $1.8-2.2 \mathrm{eV}$ region, indicating that species are kinetically related. The $1.04 \mu \mathrm{s}$ spectrum exhibits the $2.48 \mathrm{eV}(500 \mathrm{~nm})$ shoulder of ${ }^{3} \mathrm{PtOEP}$ (Figure 2), allowing the TA spectrum of ${ }^{3} \mathrm{PtOEP}$ to be recovered from the sum of the $1.04 \mu$ s and $16 \mu$ s spectra. Triplet energy transfer from ${ }^{3}$ PtOEP to ground state V79, yields ${ }^{3} \mathrm{~V} 79$, which decays over $16 \mu \mathrm{s}$. The species-associated spectra are plotted in Figure 3.

The spectrum of ${ }^{3}$ V79 hardly exhibits a bleach at all, which is ascribed to overlapping excited-state absorptions in the ground-state bleach region. As explained above, this is entirely consistent with the results of TD-DFT calculations. The transient absorption spectrum of ${ }^{3} \mathrm{V79}$ was calibrated from the extinction of the Q-band bleach of ${ }^{3} \mathrm{PtOEP}$ and the triplet energy transfer yield, taken from the ${ }^{3} \mathrm{PtOEP}$ state lifetimes in the presence $\left(\tau_{3}\right.$ PtOEP,q $)$ and absence of V79 ( $\tau_{3}$ PtOEP $)$.

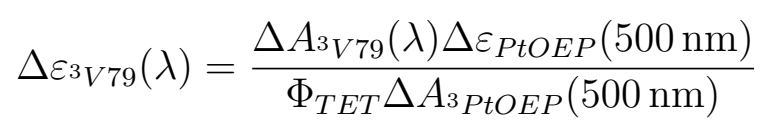



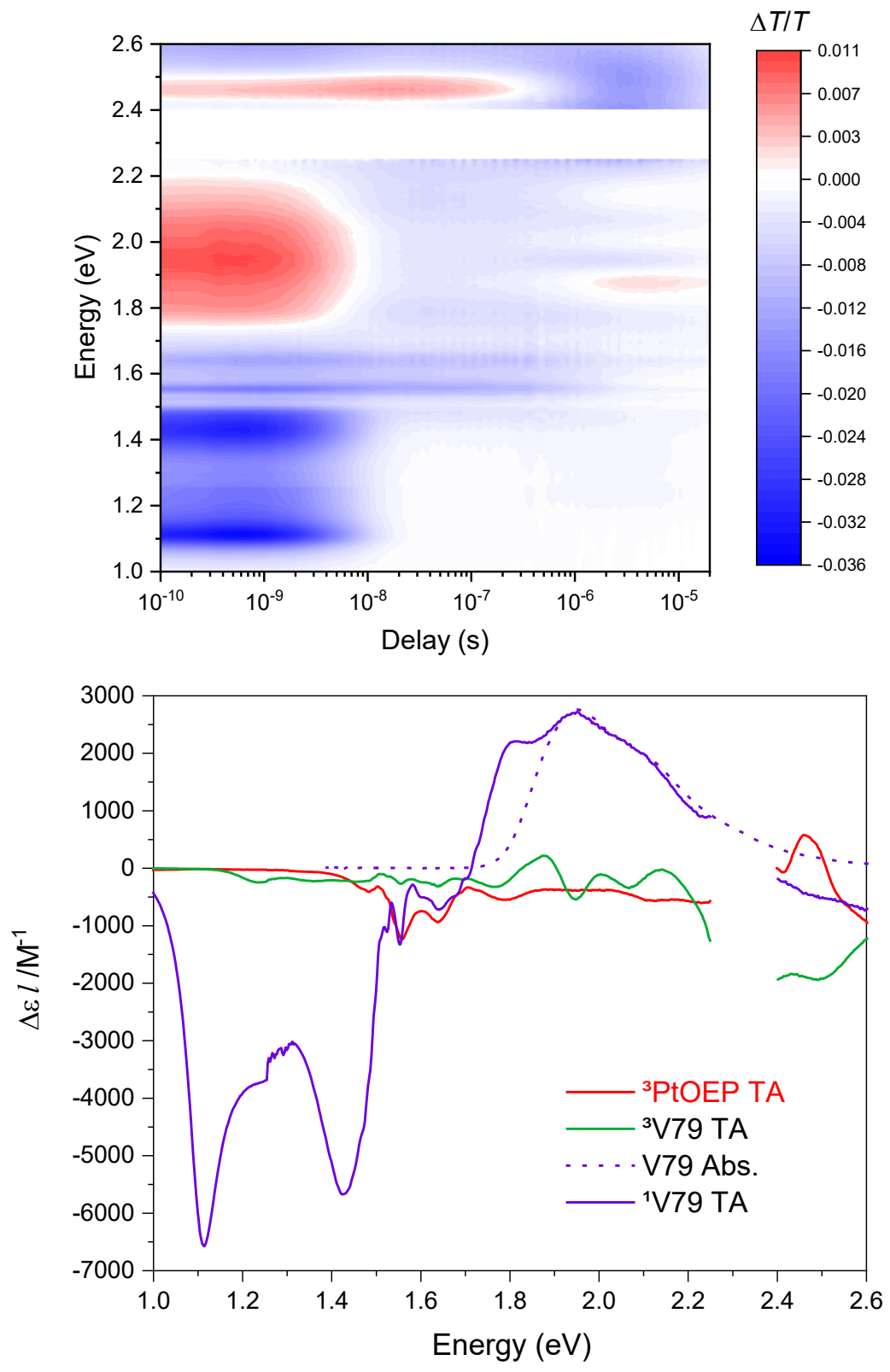

Figure 3: Top: The transient absorption spectrum of a mixture of V79 and PtOEP. Both species are excited by the $532 \mathrm{~nm}$ laser pulse, with the ${ }^{1} \mathrm{~V} 79$ decaying on a nanosecond time-scale. The excited PtOEP undergoes intersystem crossing, resulting in long-lived ${ }^{3} \mathrm{PtOEP}$ which transfers energy to ground state V79 molecules resulting in ${ }^{3} \mathrm{~V} 79$, which has a $16 \mu$ s lifetime. Bottom: The species-associated spectra. 


$$
\Phi_{T E T}=1-\frac{\tau_{3} P t O E P, q}{\tau_{3} P t O E P}
$$

\subsection{Exciton dynamics in a V79 film}

The TA spectrum of a drop-cast film of V79 is shown in Figure 4. There is a rapid decay evident on the few ps timescale, $\mathrm{a} \approx 100$ ps-lifetime species with a broad ground-state bleach, and a long-lived species with a narrow, red-shifted ground state bleach. The decay-associated spectra are shown at the bottom of Figure 4. There was no discernable effect on the observed emission due to magnetic fields (Figure 4). Reasons for this are discussed below.

\section{Discussion}

To interpret the decay-associated spectra in terms of species, we require a kinetic model. There are two distinct situations: a branching whereby an initial photo-generated species gives rise to two subsequent species in parallel, and a sequential model, where there is a linear progression of energy from one species to another via an intermediate.

If the spectra with $3.35 \mathrm{ps}, 101 \mathrm{ps}$ and $5.5 \mathrm{~ns}$ decays are denoted $\sigma_{n}(n=1,2,3)$, then the expected species-associated spectra, $\sigma_{\alpha}(\alpha=A, B, C)$ are in the sequential case,

$$
\begin{array}{r}
\sigma_{A}=\sigma_{1}+\sigma_{2}+\sigma_{3} \\
\sigma_{B_{s e q .}}=\sigma_{2}+\sigma_{3} \\
\sigma_{C}=\sigma_{3}
\end{array}
$$

In the parallel case, only $\sigma_{B}$ is different,

$$
\sigma_{B_{\|}}=\sigma_{2}
$$



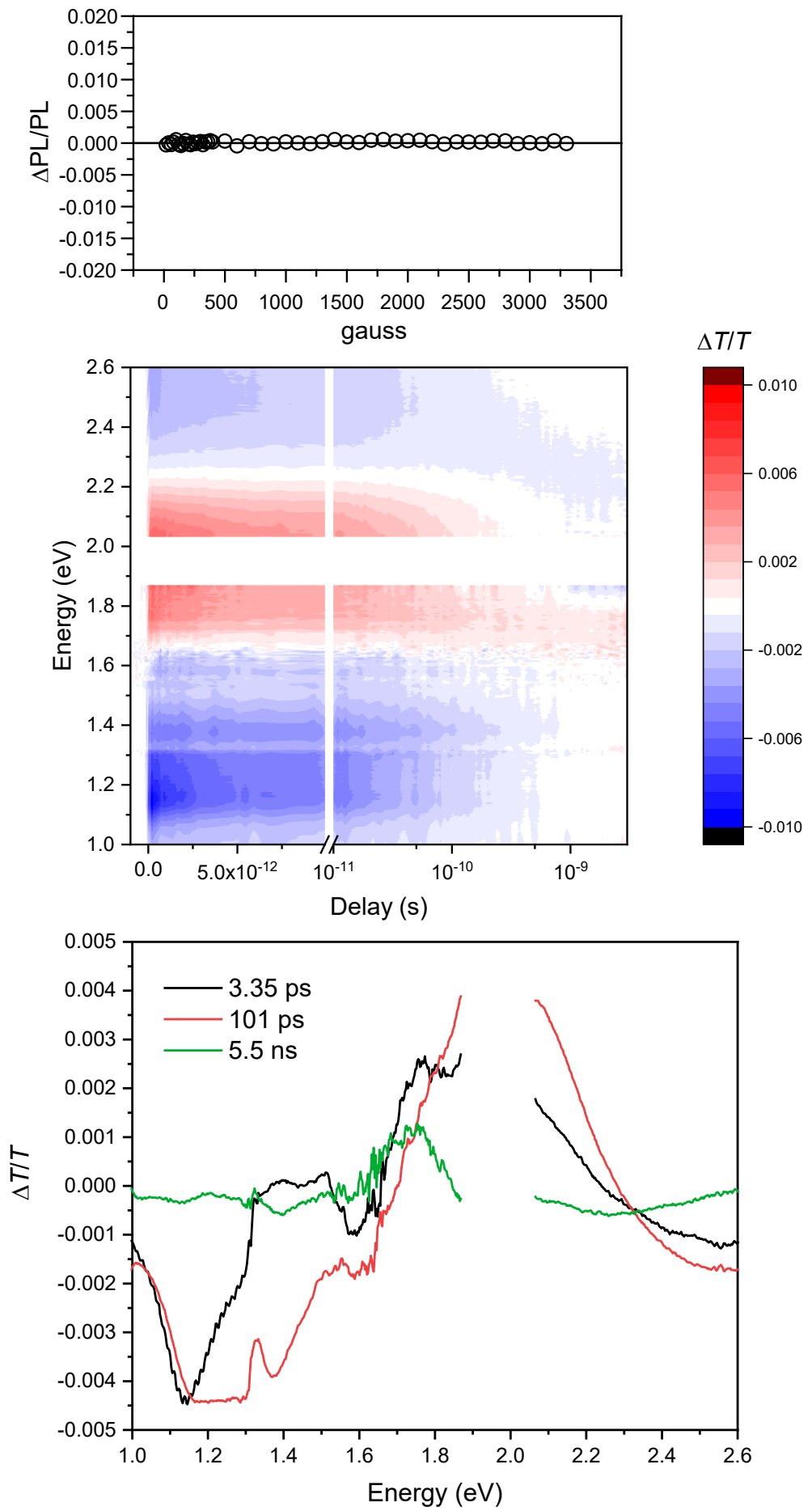

Figure 4: Top: Film luminescence as a function of magnetic field strength. Middle: The transient absorption of a drop-cast film of V79. Bottom: The decay-associated spectra. 


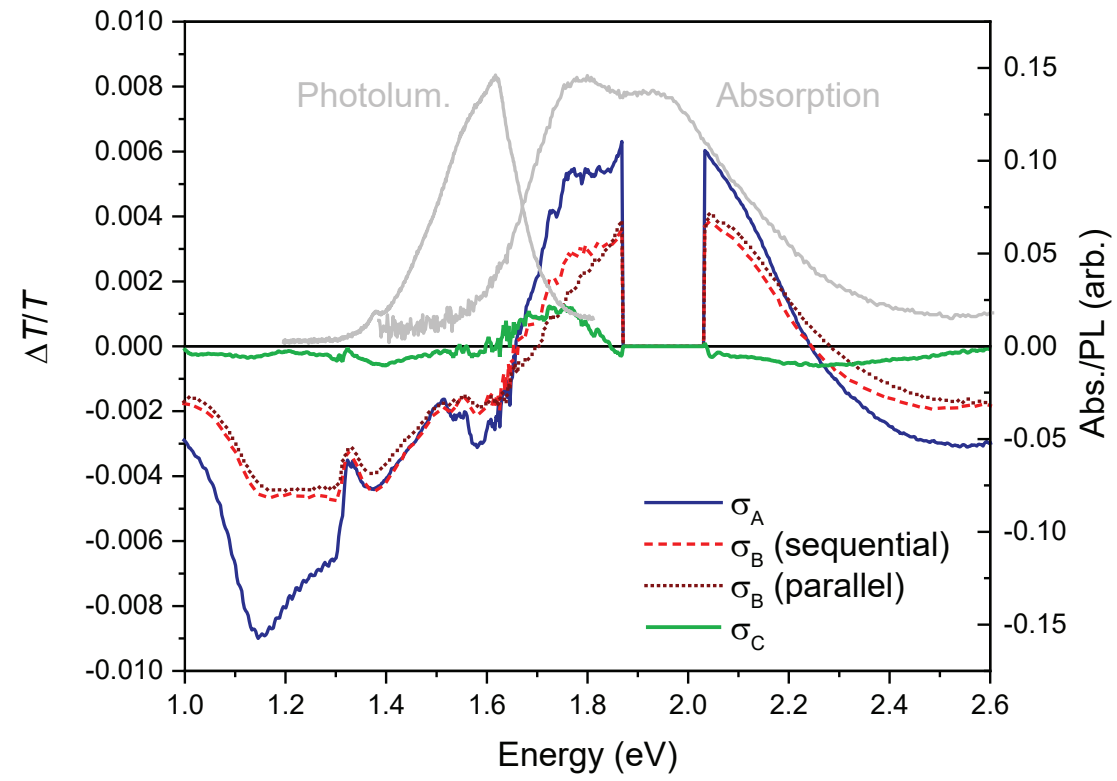

Figure 5: The species-associated spectra extracted from the data in Figure 4. 
The species associated spectra are shown in Figure 5.

There is no difference in $\sigma_{A}$ or $\sigma_{C}$ in the models. It is expected that the initially prepared state will be the $S_{1}$ state of V79. Comparing $\sigma_{A}$ to the transient V79 spectrum in Figure 2, we see that the bleach and the strong $1.1 \mathrm{eV}$ excited-state absorption are accounted for. The absorption spectrum of the film, shown in Figure 5, is red-shifted compared to the solution, indicating excitonic coupling. Any stimulated emission feature is probably overlapped by an excited-state absorption.

It is tempting to attribute the final species represented by $\sigma_{C}$ to the ${ }^{3} \mathrm{~V} 79$ triplet. This could result from singlet fission of the ${ }^{1}$ V79 state, and as shown in Figure 3, the triplet has extensive excited-state absorptions which mask the ground state bleach. Excitonic coupling in the solid could shift these absorptions compared to solution, resulting in a transient spectrum that differs. The small bleach rises from $1.55 \mathrm{eV}$, matching the low energy edge of the film absorption spectrum.

The possibility that a charge-transfer state is generated is not supported by calculations. A TD- $\omega$ B97X-D/6-311G(d)// $\omega$ B97X-D/6-31G(d) calculation of the V79 cation predicts a strong excitation $(f=0.7)$ at $2.0 \mathrm{eV}$. Given that the $S_{1}-S_{0}$ transition was calculated too high by some $0.6 \mathrm{eV}$, it would be expected that the cation would absorb to lower energy than the calculated $2.0 \mathrm{eV}$ and be evident in the transient absorption spectrum. Nevertheless, we note that films of violanthrone itself, which lacks the solubilizing octyloxy groups, photoconductivity is observed.[34] Attempts to observed photoconductivity in V79 were unsuccessful.

The choice of a sequential or parallel models comes down to a choice of $\sigma_{B}$ spectrum. The sequential model appears to have a bleach which is the same shape as that in $\sigma_{A}$, closely matching the absorption spectrum. Conversely, the parallel $\sigma_{B}$ spectrum has a diminished bleach around $1.75 \mathrm{eV}$. Another point in favour of the sequential model is the rate for generation of the putative triplet. It is expected that singlet fission is slightly endothermic in this case. As such, 
a singlet lifetime of 3 ps would be unlikely. In the case of tetracene crystals, another endothermic fission system, the lifetime is hundreds of ps,[35] and even in films where the decay is to crystal defects, the decay is on the order of $100 \mathrm{ps} .[36,37]$ We thus attribute spectrum $\sigma_{B}$ to an excitonically relaxed singlet which gives rise to triplets by endothermic singlet fission.

Magnetic fields are often used as a method to characterize singlet fission. It is well established that a pool of statistically populated triplets undergo inhibited triplet-triplet annihilation in magnetic fields, the effect usually being of the order of several percent at fields of $3000 \mathrm{G}$.[38] By the principle of detailed balance, one would therefore also expect the singlet fission process to produce a pool of statistical triplets to be hindered, and thus potentially enhanced photoluminescence would result. This has been observed in solution[39] and in the solid state.[3, 40]

However, a magnetic field enhancement of PL requires a quasi-equilibrium between the bright singlet state and the weakly-coupled triplet pair $((T \ldots T))$.[41] This requires that the rate for reformation of the singlet state is comparable to or exceeds that of triplet dissociation (or decorrelation). The lack of a magnetic field effect here suggests that either this criteria is missing, or that singlet fission is not occuring. Since singlet fission here would be slightly endothermic, it is expected that population could indeed be exchanged between the $S_{1}$ and $(T \ldots T)$ states. Tetracene, which is an endothermic singlet fission material, exhibits the characteristic effect.[3] However, if the dissociation of the $(T \ldots T)$ state is rapid, all photoluminescence is detected on the first pass, and there would be no magnetic field effect observed. It may thus be concluded that the absence of a magnetic field effect does not rule out singlet fission in the same way that its presence would confirm it. No delayed fluorescence was observed.

It was noted that the fluorescence lifetime in the closely related V78 chromophore was as short as 20 ps.[25] V78 differs by having ester linkages where V79 has ether groups. It is thus likely that V78 also decays to a (pair of) triplet states by singlet fission. This underscores a general problem with performing triplet fusion upconversion in the condensed phase at finite 
temperature. Even where singlet fission is endothermic, it likely has a rate that is faster than fluorescence, and is thus detrimental to the desired outcome. While this problem can be mitigated by doping, a more elegant approach would be to engineer the electronic coupling between chromophores to tune the singlet fission (and thus also triplet fusion) rate well below the fluorescence rate. Since the triplet lifetime exceeds the fluorescence lifetime by orders of magnitude, such an approach would seem plausible.

\section{Conclusions}

The transient absorption spectra of V79 and PtOEP were studied separately, and used determine that the transient absorption spectrum of ${ }^{3} V 79$ has a ground-state bleach which is largely balanced by excited state absorption. This was supported by the results of time-dependent density functional theory.

In V79 films, the excited singlet state is found to live only about 100 ps before decaying to a triplet state, as evidenced by the lack of a strong ground-state bleach and the longevity

of the terminal electronic state. The absence of intersystem crossing identified in solution and the demonstration that V79 undergoes TTA strongly suggest that in the film it is undergoing endothermic singlet fission. Attempts to conclusively show this with magnetic field effects were unsuccessful.

\section{Data Availability}

The data that support the findings of this study are available from the corresponding author upon reasonable request. 


\section{Acknowledgements}

This work was supported by the Australian Research Council (Centre of Excellence in Exciton

Science CE170100026). It was undertaken with the assistance of resources from the National Computational Infrastructure (NCI), which is supported by the Australian Government (Project zu57).

\section{References}

[1] Schulze, T. F. \& Schmidt, T. W. Photochemical upconversion: present status and prospects for its application to solar energy conversion. Ener. Environ. Sci. 8, 103-125 (2015).

[2] Zhou, Y., Ruchlin, C., Robb, A. J. \& Hanson, K. Singlet sensitization-enhanced upconversion solar cells via self-assembled trilayers. ACS Energy Lett. 4, 1458-1463 (2019).

[3] Einzinger, M. et al. Sensitization of silicon by singlet exciton fission in tetracene. Nature 571, 90-94 (2019).

[4] MacQueen, R. W. et al. Crystalline silicon solar cells with tetracene interlayers: the path to silicon-singlet fission heterojunction devices. Mater. Horiz. 5, 1065-1075 (2018).

[5] Rao, A. \& Friend, R. H. Harnessing singlet exciton fission to break the shockley-queisser limit. Nat. Rev. Mater. 2, 17063 (2017).

[6] Tayebjee, M. J. Y., McCamey, D. R. \& Schmidt, T. W. Beyond shockley-queisser: Molecular approaches to high-efficiency photovoltaics. J. Phys. Chem. Lett. 6, 2367-2378 (2015).

[7] Jiang, Y. et al. Singlet fission and tandem solar cells reduce thermal degradation and enhance lifespan. Prog. Photovolt. (2021). 
[8] Ravetz, B. D. et al. Photoredox catalysis using infrared light via triplet fusion upconversion. Nature 565, 343-346 (2019).

[9] Khnayzer, R. S. et al. Upconversion-powered photoelectrochemistry. Chem. Commun. 48, 209-211 (2012).

[10] Bonnet, S. Shifting the light activation of metallodrugs to the red and near-infrared region in anticancer phototherapy. Comm. Inorg. Chem. 35, 179-213 (2015).

[11] Zhou, J., Liu, Q., Feng, W., Sun, Y. \& Li, F. Upconversion luminescent materials: Advances and applications. Chem. Rev. 115, 395-465 (2015).

[12] Wen, S. et al. Future and challenges for hybrid upconversion nanosystems. Nat. Photon. 13, 828-838 (2019).

[13] Pedrini, J. \& Monguzzi, A. Recent advances in the application triplet-triplet annihilationbased photon upconversion systems to solar technologies. J. Photon. Ener. 8, $8-8-16$ (2017).

[14] Schmidt, T. W. \& Castellano, F. N. Photochemical upconversion: the primacy of kinetics. J. Phys. Chem. Lett. 5, 4062-4072 (2014).

[15] Bharmoria, P., Bildirir, H. \& Moth-Poulsen, K. Triplet-triplet annihilation based near infrared to visible molecular photon upconversion. Chem. Soc. Rev. 49, 6529-6554 (2020).

[16] Smith, M. B. \& Michl, J. Singlet fission. Chem. Rev. 110, 6891-6936 (2010).

[17] Smith, M. B. \& Michl, J. Recent advances in singlet fission. Ann. Rev. Phys. Chem. 64, 361-386 (2013). 
[18] Congreve, D. N. et al. External quantum efficiency above $100 \%$ in a singlet-excitonfission-based organic photovoltaic cell. Science 340, 334-337 (2013).

[19] Ehrler, B., Wilson, M. W. B., Rao, A., Friend, R. H. \& Greenham, N. C. Singlet exciton fission-sensitized infrared quantum dot solar cells. Nano Letters 12, 1053-1057 (2012).

[20] Tritsch, J. R., Chan, W.-L., Wu, X., Monahan, N. R. \& Zhu, X. Harvesting singlet fission for solar energy conversion via triplet energy transfer. Nature Commun. 4, 2679 (2013).

[21] Jadhav, P. J., Mohanty, A., Sussman, J., Lee, J. \& Baldo, M. A. Singlet exciton fission in nanostructured organic solar cells. Nano Letters 11, 1495-1498 (2011).

[22] Tayebjee, M. J. Y., Gray-Weale, A. A. \& Schmidt, T. W. Thermodynamic limit of exciton fission solar cell efficiency. J. Phys. Chem. Lett. 3, 2749-2754 (2012).

[23] Fückel, B. et al. Singlet oxygen mediated photochemical upconversion of nir light. $J$. Phys. Chem. Lett. 2, 966-971 (2011).

[24] Gholizadeh, E. M. et al. Photochemical upconversion of near-infrared light from below the silicon bandgap. Nat. Photonics 14, 585-590 (2020).

[25] Kakuichi, M., Kasatani, K. \& Morita, Y. Preparation of nanoparticles of a violanthrone derivative with properties of j-like aggregates. Trans. Mat. Res. Soc. Japan 37, 471-474 (2012).

[26] Wu, M. et al. Solid-state infrared-to-visible upconversion sensitized by colloidal nanocrystals. Nat. Photonics 10, 31-34 (2016).

[27] Wieghold, S., Bieber, A. S., Van Orman, Z. A., Rodriguez, A. \& Nienhaus, L. Is disorder beneficial in perovskite-sensitized solid-state upconversion? the role of dbp doping in rubrene. J. Phys. Chem. C 124, 18132-18140 (2020). 
[28] Penconi, M., Gentili, P. L., Massaro, G., Elisei, F. \& Ortica, F. A triplet-triplet annihilation based up-conversion process investigated in homogeneous solutions and oil-in-water microemulsions of a surfactant. Photochem. Photobiol. Sci. 13, 48-61 (2014).

[29] Gao, C. et al. Intramolecular versus intermolecular triplet fusion in multichromophoric photochemical upconversion. J. Phys. Chem. C 123, 20181-20187 (2019).

[30] Guse, J. A. et al. Spectral dependence of direct and trap-mediated recombination processes in lead halide perovskites using time resolved microwave conductivity. Phys. Chem. Chem. Phys. 18, 12043-12049 (2016).

[31] Frisch, M. J. et al. Gaussian 16 RevisionC.01 (2016). Gaussian Inc. Wallingford CT.

[32] Chai, J.-D. \& Head-Gordon, M. Long-range corrected hybrid density functionals with damped atom-atom dispersion corrections. Phys. Chem. Chem. Phys. 10, 6615-6620 (2008).

[33] Krishnan, R., Binkley, J. S., Seeger, R. \& Pople, J. A. Self-consistent molecular orbital methods. XX. a basis set for correlated wave functions. J. Chem. Phys. 72, 650-654 (1980).

[34] Akamatu, H. \& Inokuchi, H. Photoconductivity of violanthrone. J. Chem. Phys. 20, 1481-1483 (1952).

[35] Piland, G. B. \& Bardeen, C. J. How morphology affects singlet fission in crystalline tetracene. J. Phys. Chem. Lett. 6, 1841-1846 (2015).

[36] Burdett, J. J., Gosztola, D. \& Bardeen, C. J. The dependence of singlet exciton relaxation on excitation density and temperature in polycrystalline tetracene thin films: Kinetic ev- 
idence for a dark intermediate state and implications for singlet fission. J. Chem. Phys. 135, 214508 (2011).

[37] Tayebjee, M. J. Y., Clady, R. G. C. R. \& Schmidt, T. W. The exciton dynamics in tetracene thin films. Phys. Chem. Chem. Phys. 15, 14797-14805 (2013).

[38] Merrifield, R. E. Theory of magnetic field effects on the mutual annihilation of triplet excitons. J. Chem. Phys. 48, 4318-4319 (1968).

[39] Thompson, N. J., Hontz, E., Chang, W., Van Voorhis, T. \& Baldo, M. Magnetic field dependence of singlet fission in solutions of diphenyl tetracene. Phil. Trans. Roy. Soc. A 373 (2015).

[40] Groff, R., Avakian, P. \& Merrifield, R. Magnetic field dependence of delayed fluorescence from tetracene crystals. J. Luminesc. 1-2, 218 - 223 (1970).

[41] Burdett, J. J., Piland, G. B. \& Bardeen, C. J. Magnetic field effects and the role of spin states in singlet fission. Chem. Phys. Lett. 585, 1-10 (2013). 